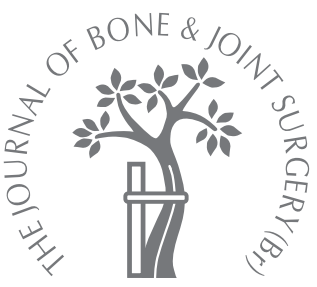

\title{
Comparison of polyethylene wear in anatomical and reversed shoulder prostheses
}

\author{
A. Terrier, \\ F. Merlini, \\ D. P. Pioletti, \\ A. Farron \\ From École \\ Polytechnique \\ Fédérale de \\ Lausanne, Lausanne, \\ Switzerland
}

\begin{abstract}
Wear of polyethylene is associated with aseptic loosening of orthopaedic implants and has been observed in hip and knee prostheses and anatomical implants for the shoulder. The reversed shoulder prostheses have not been assessed as yet. We investigated the volumetric polyethylene wear of the reversed and anatomical Aequalis shoulder prostheses using a mathematical musculoskeletal model. Movement and joint stability were achieved by EMG-controlled activation of the muscles. A non-constant wear factor was considered. Simulated activities of daily living were estimated from in vivo recorded data.

After one year of use, the volumetric wear was $8.4 \mathrm{~mm}^{3}$ for the anatomical prosthesis, but $44.6 \mathrm{~mm}^{3}$ for the reversed version. For the anatomical prosthesis the predictions for contact pressure and wear were consistent with biomechanical and clinical data. The abrasive wear of the polyethylene in reversed prostheses should not be underestimated, and further analysis, both experimental and clinical, is required.
\end{abstract}

Wear of polyethylene is a recurrent problem in joint replacement. In addition to the permanent deformation or complete destruction of the component, small particles of polyethylene can cause an inflammatory response in the surrounding bone. ${ }^{1-3}$ The size of these particles, which depends on the design of the prosthesis, has been shown to be an important factor in the cellular reaction. ${ }^{4,5}$ Particles of debris generated by wear are also responsible for changes in osteogenesis, ${ }^{6-8}$ producing an imbalance between the osteoclastic and osteoblastic activity which may result in resorption of bone at the implant-bone interface, leading to failure of the bond between the implant and the host bone.

Extensive investigation has been undertaken of polyethylene wear in hip and knee prostheses, but less attention has been paid to this with shoulder replacements. In the anatomical total shoulder replacement, failure of the polyethylene component is the major cause of complications related to periprosthetic osteolysis and aseptic loosening. ${ }^{9,10}$ The presence of particles of polyethylene debris arising from abrasive wear are thought to be one of the main causes of glenoid loosening. ${ }^{9,11}$ The polyethylene wear of anatomical prostheses has been observed clinically and in retrieved glenoid components. ${ }^{12-14}$ It has been shown that various types of wear are present, but abrasion was found to be more important than pitting and delamination due to fatigue. ${ }^{13}$ The location of the wear was not statistically different in the retrieved glenoid, but a new conforming surface was centered superiorly. ${ }^{12} \mathrm{~A}$ similar pattern of wear has been observed in a finiteelement study. ${ }^{15}$

Since approval by the United States Food and Drug Administration in 2004, ${ }^{16}$ reversed prostheses have been increasingly used for glenohumeral arthropathy associated with partial or severe deficiency of the rotator cuff. ${ }^{10,17}$ The medialised and semi-constrained artificial joint restores stability and loss of movement when muscles of the rotator cuff are deficient. The glenohumeral force has been estimated to be reduced by half in a reversed prosthesis compared with an anatomical model. ${ }^{18}$ Also, since the articular surfaces of a reversed prosthesis are more congruent than those of the anatomical model, the contact pressure should also be much lower. However, polyethylene wear in reversed prostheses is not trivial and the volume of wear particles has been shown to be greater at lower contact pressure, for larger contact surfaces and with larger sliding distances. ${ }^{19-23}$ Until now, problems with wear have mainly been related to the scapular notch, ${ }^{24}$ but abrasive wear of the humeral component may also be an issue.

Our aim was to obtain a preliminary estimation of the abrasive wear of the polyethylene component of reversed prostheses. We used a mathematical musculoskeletal model 

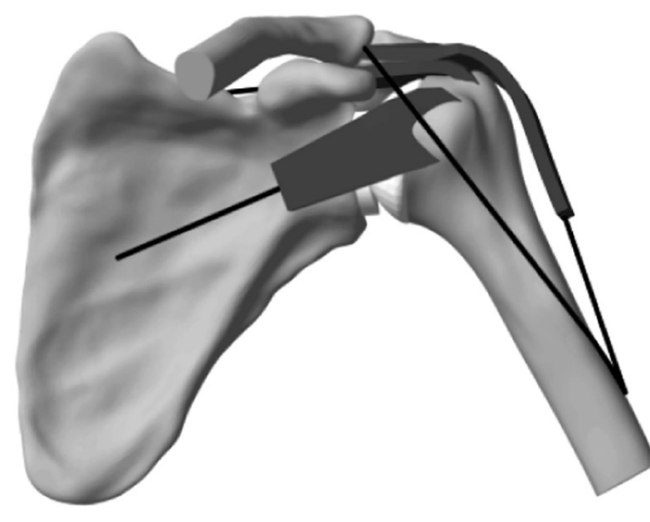

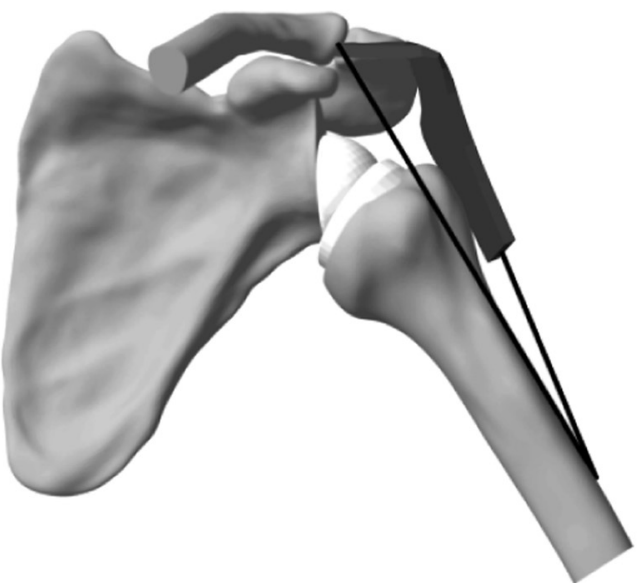

Fig. 1

Diagrams showing the location of the strip-like parts of the muscles (dark grey) and the active cable-like part (black). The reversed prosthesis (right) is shown without any rotator-cuff muscle.

of the shoulder to estimate and to compare the volume of abrasive wear of polyethylene for a reversed prosthesis and for an anatomical prosthesis during the activities of daily living over a period of one year. We wished to test the hypothesis that the volume of polyethylene wear particles was as important in a reversed prosthesis as in an anatomical model.

\section{Materials and Methods}

Our comparative analysis was performed using a mathematical musculoskeletal model of the glenohumeral joint, ${ }^{25,26}$ which has already been used to estimate the contact force in reversed prostheses. ${ }^{18}$ The model includes the scapula, the proximal part of the humerus and six scapulohumeral muscles: the middle deltoid, the anterior deltoid, the posterior deltoid, supraspinatus, subscapularis and infraspinatus combined with teres minor. The geometry of the bones was reconstructed from CT images of a cadaver scapula, and the muscle attachments were measured from dissection of the same scapula. The bones were rigid, while the muscles were deformable and could contract. Abduction was carried out in the scapular plane by activation of the muscles estimated by electromyography (EMG) and controlled by a synchronisation algorithm. Stability of the glenohumeral joint was also achieved by muscles allowing for the natural translations of the humeral head. The rotation of the scapula was included through a scapulohumeral movement ratio of $2: 1$. The weight of the arm was $3.75 \mathrm{~kg}$, corresponding to $5 \%$ of the body-weight.

We used the reversed and the anatomical Aequalis prosthesis (Tornier Inc, Edina, Minnesota). For the reversed prosthesis, the diameter of the glenoid component was $36 \mathrm{~mm}$ and the humeral component was perfectly congruent. For the anatomical prosthesis, the diameter of the head of the humerus was $48 \mathrm{~mm}$ and the radial mismatch was $6 \mathrm{~mm}$, with the radius of curvature of the glenoid being $30 \mathrm{~mm}$. For both prostheses, the polyethylene component was ultra-high molecular weight (UHMWPE), raw extruded from GUR 1020 resins, gamma-sterilised and not cross-linked. The prostheses were made from cobalt-chrome (Co-Cr) alloy. The geometrical description of each component was obtained from the manufacturer (Tornier Inc). The prostheses were inserted into the musculoskeletal model according to the recommendation of the manufacturer using solidworks computer-aided design software (Dassault Systèmes, Concord, Massachusetts). The positioning of each component was assessed by a senior orthopaedic surgeon (AF). In the mathematical analysis, the metal parts were assumed to be rigid, while the polyethylene components were elastic $(\mathrm{E}$ (elastic modulus $)=500 \mathrm{MPa}, \mathrm{v}($ Poisson ratio $)=0.4)$. For the reversed prosthesis, the muscles of the rotator cuff were deactivated, since this prosthesis is used when these muscles are partly or completely deficient (Fig. 1).

The linear wear was estimated from Archard's law ${ }^{27}$ which states that $\mathrm{H}=\mathrm{k} \mathrm{p} \mathrm{s}$, where $\mathrm{k}$ is the wear factor, $\mathrm{p}$ the contact pressure and $\mathrm{s}$ the sliding distance. Since a large pressure variation was expected between the two prostheses, the pressure dependency on the wear factor was considered, based on experimental measurements. ${ }^{19}$ Contact pressure and sliding distance were obtained from the musculoskeletal model. Different movements were considered. For each movement $(\mathrm{m})$, the wear at some point of the articular surface was $H_{m}=\sum k\left(p_{j}\right) p_{j} s_{j}$, corresponding to the sum of small sliding distances. The daily linear wear $\left(\mathrm{H}_{\mathrm{d}}=\sum \mathrm{N}_{\mathrm{m}} \mathrm{H}_{\mathrm{m}}\right)$ was the sum of each movement, $H_{m}$ wear, weighted by their respective daily frequency, $\mathrm{N}_{\mathrm{m}} \cdot \mathrm{H}_{\mathrm{d}}$ corresponded to the superposition of different movements, each one being performed $\mathrm{N}_{\mathrm{m}}$ times per day. The yearly linear wear was then $\mathrm{H}_{\mathrm{y}}=365 \mathrm{H}_{\mathrm{d}}$. Finally, the volumetric wear was calculated as the 
Table I. The daily frequency of eight levels of abduction $\left(^{\circ}\right)$ were estimated from recorded kinematics collected by a portable device on 31 volunteers during activities of daily living for eight hours ${ }^{26}$

\begin{tabular}{lllllllll}
\hline Abduction level & $\mathbf{1 0}$ & $\mathbf{3 0}$ & $\mathbf{5 0}$ & $\mathbf{7 0}$ & $\mathbf{9 0}$ & $\mathbf{1 1 0}$ & $\mathbf{1 3 0}$ & $\mathbf{1 5 0}$ \\
\hline Daily frequency & 13021 & 6790 & 5557 & 2678 & 633 & 153 & 34 & 37 \\
\hline
\end{tabular}

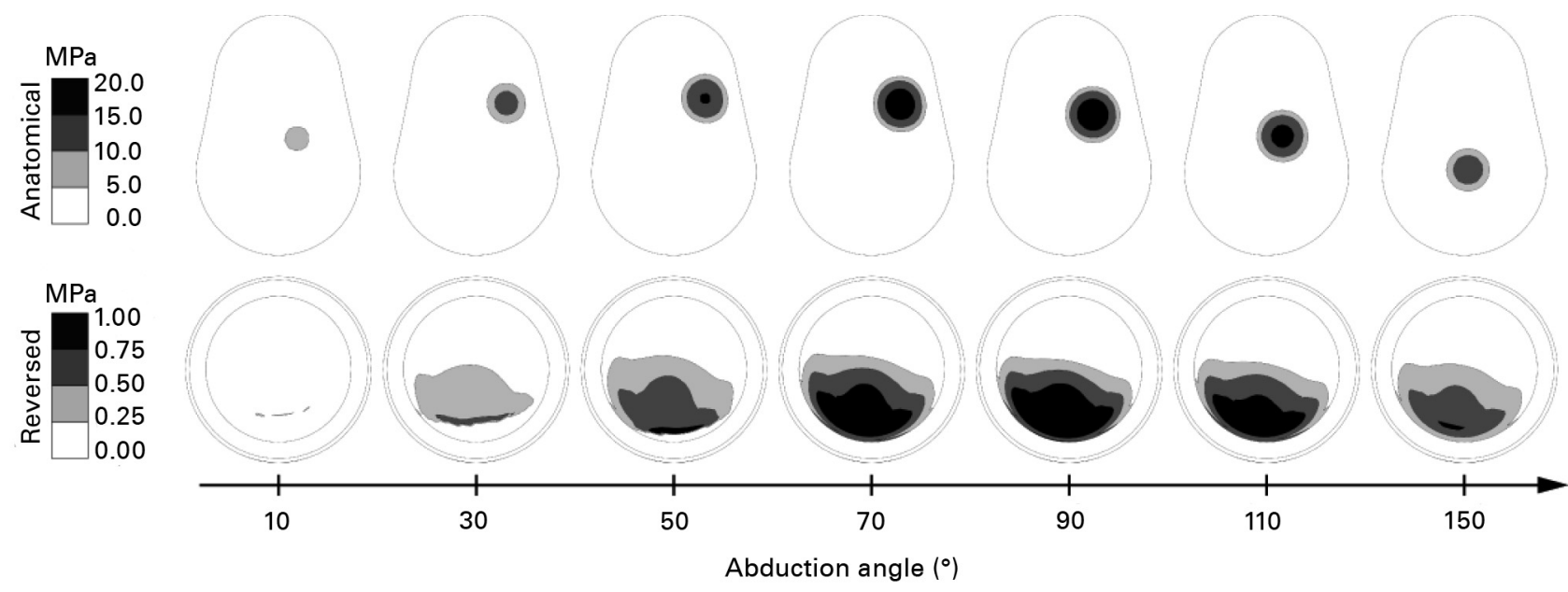

Fig. 2

Diagrams showing the patterns of contact pressure on the polyethylene surface for every $10^{\circ}$ of abduction for the anatomical and reversed prostheses.

difference in volume between the original component and the worn component.

In order to reproduce the wear of the activities of daily living for one day, $M$ abduction levels at a frequency of $\mathrm{N}_{\mathrm{m}}$ were considered. The abduction levels and corresponding frequencies were estimated by recording the shoulder kinematics on 31 volunteers during activities of daily living for eight hours, using an ambulatory system of inertial captors. ${ }^{28}$ The entire recorded kinematics were analysed and summarised in different levels of arm elevation, which were associated to a specific hourly frequency. In our study we considered eight levels of abduction. The daily frequency $\mathrm{N}_{\mathrm{m}}$ of each movement was estimated assuming 12 hours of activity per day (Table I).

All mathematical analyses were performed using the integrated finite element analysis program Simulia Abaqus (Dassault Systèmes). The deformable parts were meshed with linear hexahedral elements. The glenohumeral contact pressure was solved by the standard hard contact method for large sliding, without friction. The linear wear was calculated by an external programming language (Python script; Python Software Foundation, Hampton, New Hampshire), directly reading the Abaqus output of each simulated cycle. Within the same Python script, each point of the original polyethylene surface was then displaced by its amount of linear wear.

\section{Results}

The glenohumeral contact pressure on the polyethylene surface was different for each design (Fig. 2). For the anatomical prosthesis, the contact pattern moved from the inferior to the superior side of the surface of the glenoid during the first $30^{\circ}$ of abduction, and returned to the inferior side during the rest of abduction. The contact pressure reached $19 \mathrm{MPa}$ at $90^{\circ}$ of abduction. For the reversed prosthesis, the contact pattern on the surface of the humeral component remained at the inferior side during the entire range of abduction. The contact pressure reached $1.2 \mathrm{MPa}$ at $90^{\circ}$ of abduction. Overall, the contact pressure was approximately 20 times lower for the reversed than for the anatomical prosthesis.

After one year of simulated activity, the linear wear of the polyethylene was small in each design (Fig. 3). The maximum depth of wear reached $0.2 \mathrm{~mm}$ on the glenoid component of the anatomical prosthesis and did not exceed $0.13 \mathrm{~mm}$ on the humeral component of the reversed prosthesis. The volumetric wear was $8.4 \mathrm{~mm}^{3}$ for the anatomical, but $44.6 \mathrm{~mm}^{3}$ for the reversed prosthesis.

\section{Discussion}

In our study the anatomical and reversed Aequalis prostheses were tested within the same numerical musculoskeletal model, which simulated one year of activities of daily living. Our hypothesis that the polyethylene wear in reversed 


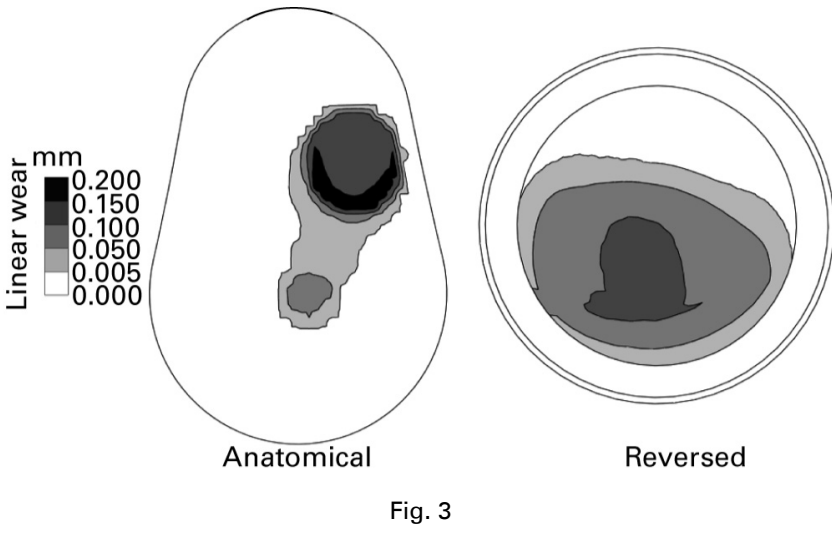

Diagram showing the linear wear of the polyethylene components after activities of daily living for one year.

prostheses would be as important as in anatomical prostheses was confirmed.

As with other joint replacements, polyethylene wear particles are found around shoulder prostheses. They have been observed around the humeral and glenoid components of anatomical shoulder prostheses removed after aseptic loosening associated with osteolysis. ${ }^{29,30}$ Analyses of retrieved glenoid components have shown that several mechanisms of wear occur with variable degrees of importance. ${ }^{12-14,31}$ In one study, from ten retrieved glenoid components, nine modes of wear were examined in the four quadrants of the articular surface. ${ }^{13}$ Surface wear was dominant, but was also combined with fatigue wear. The authors related this combination of surface and fatigue wear to that seen in hip and knee implants, in which surface and fatigue wear are respectively dominant. Another study reported that the worn surface of the glenoid component in anatomical shoulder replacements matched the radius of the humeral head perfectly and was shifted posteriorly and superiorly, ${ }^{12}$ as predicted in our model. From the initial radial mismatch and the mean time of retrieval, the maximal rate of linear wear was approximately $0.7 \mathrm{~mm} /$ year, which is consistent with the $0.2 \mathrm{~mm} /$ year predicted in our study for the anatomical design. Another review examined and quantified the wear of 20 retrieved glenoid components at revision. ${ }^{31}$ Three patterns of wear were reported: centred (abrasion and cold flow), diffuse (pitting) and rim. This study confirmed that the curvature radius of the glenoid component gradually adapts to the radius of the humeral head. A double concavity wear was observed, as noted in our simulations (Fig. 3). In addition to the above clinical studies, a mathematical parametric study predicted various levels of wear in anatomical prostheses. ${ }^{15}$ Using a maximum joint force of $406 \mathrm{~N}$, compared with $648 \mathrm{~N}$ in our study, a constant wear factor and a single cycle of loading, the predicted peak contact pressure was $11 \mathrm{MPa}$ for radial mismatch of $6 \mathrm{~mm}$, and the volumetric wear varied from $2 \mathrm{~mm}^{3}$ to $16 \mathrm{~mm}^{3}$ according to the frequency of use. Increasing the contact surface, by decreasing the radial mismatch, reduced linear but increased volumetric wear. A similar behaviour pattern was reported in another mathematical study. ${ }^{32}$ Our results are consistent with these studies.

For reversed prostheses, wear has only been associated with the scapular notch. ${ }^{24}$ There is no information about the abrasive wear caused by the sliding of the articular surfaces of reversed implants. Wear in anatomical prostheses has already been compared with that in hip and knee implants. ${ }^{29}$ This comparison may be extended to reversed prostheses. Hip prostheses, like reversed shoulder prostheses, have a higher conforming surface and lower contact stress than knee prostheses which are similar to anatomical shoulder prostheses. Within the limitation of this association between hip and reversed shoulder prostheses, our predicted linear and volumetric wear rates for the reversed prosthesis were consistent with those of clinical studies on hip prostheses. ${ }^{33}$

The fivefold increase in volumetric wear between the anatomical and reversed prosthesis does not indicate a fivefold increase in clinical failure. In the anatomical prosthesis, the main cause of failure is loosening of the glenoid component, ${ }^{34}$ which has several causes. The inflammatory reaction to wear debris may not be the most important, compared, for example, with malalignment of the components. ${ }^{35}$ For the reversed prosthesis the glenoid component has a relatively low rate of loosening. The effect of wear debris in the reversed prosthesis may rather be associated with a higher rate of loosening of the humeral stem. ${ }^{36}$

Mathematical models predicting abrasive wear usually consider a constant wear factor which, as estimated from clinical measurements or mechanical testing, varies from $10^{-9} \mathrm{~mm}^{3} / \mathrm{Nm}$ to $10^{-6} \mathrm{~mm}^{3} / \mathrm{Nm}$. This discrepancy comes from the complexity of such wear which can be affected by various factors such as the type of prosthesis, the contact pressure, the contact surface and the sliding movement. In particular, the rate of wear decreases when the contact pressure increases as has been observed in mechanical testing ${ }^{19,20,22}$ and in clinical studies. ${ }^{37}$ In our study since the contact pressure varied 20-fold, a non-constant wear factor was considered. We used an experimental relationship obtained from a study of pin-on-disk wear of UHMWPE with polished $\mathrm{Co}-\mathrm{Cr}^{19}$ in which the wear factor was related to the contact pressure from $0.1 \mathrm{MPa}$ to $20 \mathrm{MPa}$. A change in wear regime was observed above $2 \mathrm{MPa}$, which seemed to be correlated with the differences observed clinically between hip and knee prostheses. The variable wear factor measured in this experimental study ${ }^{19}$ was in good agreement with a similar previous study. ${ }^{22}$

In the ASTM F2028-00 standard for glenoid testing, ${ }^{38}$ 25 high-load $(750 \mathrm{~N})$ activities per day are required. This was recommended for laboratory tests by an experimental study. ${ }^{39}$ In the mathematical study of Hopkins et $\mathrm{al}^{15} 30^{\circ}$ steps of complete abduction were simulated at a frequency of $10 \%, 50 \%$ and $100 \%$ of 3500 cycles per day, which had been proposed previously. ${ }^{40}$ The in vivo recorded kinemat- 
ics used in our study corresponded approximately to 3000 high-load cycles per day and 1 million cycles per year.

Both prostheses were tested in the same mechanical conditions by the same musculoskeletal model. The motor and stabilising function achieved by the muscles predicted the natural translation of the humeral head of the anatomical prosthesis and the increased muscle moment arms of the reversed implants. This method also provided the contact pressure and sliding distance during the entire elevation. A non-constant wear factor was essential to predict wear within a large range of contact pressure. The use of in vivo kinematic data was also a key point in our study. Although shoulder activity was reduced to eight levels of abduction, it was an improvement compared with more simple and hypothetical estimates of shoulder activity.

Although the present mathematical predictions of wear were not formally validated experimentally, they provide a reasonable comparative analysis. The musculoskeletal model predicts that the reversed prosthesis increases the muscle moment arms, which reduce the required muscle forces and thus the resulting joint reaction force. A reduced reaction force, together with an increased articular surface, reduces the contact pressure. ${ }^{18,25,26,41}$ The wear data for the musculoskeletal model were obtained from published experimental material. ${ }^{19}$ However, the present results should be confirmed by experimental and clinical observations and it would be of value to analyse the wear of reversed prostheses more carefully.

We did not investigate the effect of variable levels of function of the rotator-cuff complex and deltoid. Only two typical situations were considered namely a fully functional rotator cuff and deltoid muscles for the anatomical prosthesis, and completely deficient rotator-cuff muscles for the reversed prosthesis. The partial deficiency of these muscles has been specifically investigated in previous studies, ${ }^{18,25,41}$ which showed a slight effect on the articular contact pressure. We may thus assume that there would be a slight effect on the wear for the same level of activities of daily living.

Our study was performed with the reversed Aequalis device (Tornier), but the conclusions should be valid for most reversed designs. This prosthesis is based on the Grammont prosthesis and is thus rather similar to the Delta design of DePuy (Warsaw, Indiana). The reverse shoulder prosthesis from Encore (Austin, Texas) and the anatomical shoulder inverse/reverse system from Zimmer (Warsaw, Indiana) have a different baseplate fixation and humeral stem, but the articular surfaces and the general biomechanics are still very similar. Even the Bayley-Walker prosthesis (Stanmore Implants, Stanmore, United Kingdom) should behave in a similar way regarding the wear of the polyethylene.

We did not fully reproduce the complexity of the wear mechanism. Cold flow and cross shear were not accounted for, but the stress level and sliding patterns would have had small effects on the simulated movements. The stress level was indeed below the plastic limit, and the sliding path was somewhat unidirectional. Wear was estimated, but the progressive removal of matter was not simulated. This assumption was reasonable for the prediction of wear for one year, but the removal of matter should be considered for longer term predictions. ${ }^{42,43}$ Examination of retrieved polyethylene components has shown that complex and destructive wear may occur in failed anatomical and reversed prostheses. In our study, the predicted wear corresponded to an ideal case without impingement, malpositioning, subluxation, soft-tissue imbalance or other clinical issue. However, this is consistent with clinical experience since most glenoid components present no sign of catastrophic wear after one year of use. As for other mathematical models, the predictions of wear of the reversed prosthesis should be compared with those of experimental testing and clinical reports. However, since this design is relatively new there are few data available. Our preliminary mathematical study should thus promote a deeper analysis of the mechanisms and amount of wear in reversed shoulder prostheses.

This study was supported by Tornier Inc. We especially thank Y. A. Ratron (Research Director, Tornier) for his help.

Although none of the authors has received or will receive benefits for personal or professional use from a commercial party related directly or indirectly to the subject of this article, benefits have been or will be received but will be directed solely to a research fund, foundation, educational institution, or other non- profit organisation with which one or more of the authors are associated.

\section{References}

1. Schmalzried TP, Jasty M, Harris WH. Periprosthetic bone loss in total hip arthroplasty: polyethylene wear debris and the concept of the effective joint space. J Bone Joint Surg [Am] 1992;74-A:849-63.

2. Margevicius KJ, Bauer TW, McMahon JT, Brown SA, Merritt K. Isolation and characterization of debris in membranes around total joint prostheses. J Bone Joint Surg $[A m]$ 1994;76-A:1664-75.

3. Purdue PE, Koulouvaris P, Potter HG, Nestor BJ, Sculco TP. The cellular and molecular biology of periprosthetic osteolysis. Clin Orthop 2007;454:251-61.

4. Ingham E, Fisher $\mathbf{J}$. The role of macrophages in osteolysis of total joint replacement. Biomaterials 2005;26:1271-86.

5. Richards L, Brown C, Stone MH, et al. Identification of nanometre-sized ultra-high molecular weight polyethylene wear particles in samples retrieved in vivo. J Bone Joint Surg [Br] 2008;90-B:1106-13.

6. Dean DD, Lohmann CH, Sylvia VL, et al. Effect of polymer molecular weight and addition of calcium stearate on response of MG63 osteoblast-like cells to UHMWPE particles. J Orthop Res 2001;19:179-86.

7. Glant TT, Jacobs JJ, Molnar G, et al. Bone resorption activity of particulatestimulated macrophages. J Bone Miner Res 1993;8:1071-9.

8. Pioletti DP, Kottelat A. The influence of wear particles in the expression of osteoclastogenesis factors by osteoblasts. Biomaterials 2004;25:5803-8.

9. Matsen FA 3rd, Clinton J, Lynch J, Bertelsen A, Richardson ML. Glenoid component failure in total shoulder arthroplasty. J Bone Joint Surg [Am]2008;90-A:885-96.

10. Ramsey ML, Getz CL, Parsons BO. What's new in shoulder and elbow surgery. J Bone Joint Surg [Am]2008;90-A:677-87.

11. Mabrey JD, Afsar-Keshmiri A, Engh GA, et al. Standardized analysis of UHMWPE wear particles from failed total joint arthroplasties. J Biomed Mater Res 2002;63:475-83.

12. Hertel R, Ballmer FT. Observations on retrieved glenoid components. J Arthroplasty 2003;18:361-6.

13. Gunther SB, Graham J, Norris TR, Ries MD, Pruitt L. Retrieved glenoid components: a classification system for surface damage analysis. J Arthroplasty 2002;17:95-100.

14. Scarlat MM, Matsen FA 3rd. Observations on retrieved polyethylene glenoid components. J Arthroplasty 2001;16:795-801.

15. Hopkins AR, Hansen UN, Amis AA, et al. Wear in the prosthetic shoulder: association with design parameters. J Biomech Eng 2007;129:223-30.

16. No authors listed. US food and Drug administration http://www.fda.gov (date last accessed 8 June 2009). 
17. Boileau P, Watkinson D, Hatzidakis AM, Hovorka I. Neer Award 2005: The Grammont reverse shoulder prosthesis: results in cuff tear arthritis, fracture sequelae, and revision arthroplasty. J Shoulder Elbow Surg 2006;15:527-40.

18. Terrier A, Reist A, Merlini F, Farron A. Simulated joint and muscle forces in reversed and anatomic shoulder prostheses. J Bone Joint Surg [Br] 2008;90-B:751-6.

19. Saikko V. Effect of contact pressure on wear and friction of ultra-high molecular weight polyethylene in multidirectional sliding. Proc Inst Mech Engh $[\mathrm{H}]$ 2006;220:723-31

20. Barbour PSM, Barton DC, Fisher J. The influence of contact stress on the wear of Uhmwpe for total replacement hip prostheses. Wear 1995;181:250-7.

21. Mazzucco D, Spector M. Effects of contact area and stress on the volumetric wea of ultrahigh molecular weight polyethylene. Wear 2003;254:514-22.

22. Vassiliou K, Unsworth A. Is the wear factor in total joint replacements dependent on the nominal contact stress in ultra-high molecular weight polyethylene contacts? Proc Inst Mech Eng [H] 2004;218:101-7.

23. Mazzucco D, Spector M. Contact area as a critical determinant in the tribology of metal-on-polyethylene total joint arthroplasty. J Tribology 2006;128:113-21.

24. Boileau P, Watkinson DJ, Hatzidakis AM, Balg F. Grammont reverse prosthesis design, rationale, and biomechanics. J Shoulder Elbow Surg 2005;14(Suppl S):147-61

25. Terrier A, Reist A, Vogel A, Farron A. Effect of supraspinatus deficiency on humerus translation and glenohumeral contact force during abduction. Clin Biomech (Bristol, Avon) 2007;22:645-51.

26. Terrier A, Vogel A, Capezzali M, Farron A. An algorithm to allow humerus trans lation in the indeterminate problem of shoulder abduction. Med Eng Phys 2008;30:710-16.

27. Archard JF. Contact and rubbing of flat surfaces. J Appl Phys 1953;24:981-8.

28. Coley B, Jolles BM, Farron A, Aminian K. Arm position during daily activity. Gait Posture 2008;28:581-7.

29. Wirth MA, Agrawal CM, Mabrey JD, et al. Isolation and characterization of poly ethylene wear debris associated with osteolysis following total shoulder arthroplasty. J Bone Joint Surg [Am] 1999;81-A:29-37.

30. Klimkiewicz JJ, lannotti JP, Rubash HE, Shanbhag AS. Aseptic loosening of the humeral component in total shoulder arthroplasty. J Shoulder Elbow Surg 1998;7:422-6.
31. Braman JP, Falicov A, Boorman R, Matsen FA 3rd. Alterations in surface geometry in retrieved polyethylene glenoid component. J Orthop Res 2006;24:1249-60

32. Swieszkowski W, Bersee HEN, Kurzydlowski KJ. Experimental and numerical investigation of wear in the total shoulder arthroplasty. J Biomechanics 2006;39(Suppl 1):143.

33. Devane PA, Horne JG, Martin K, Coldham G, Krause B. Three-dimensional polyethylene wear of a press-fit titanium prosthesis: factors influencing generation of polyethylene debris. J Arthroplasty 1997;12:256-66.

34. Matsen FA 3rd, Clinton J, Lynch J, Bertelsen A, Richardson ML. Glenoid component failure in total shoulder arthroplasty. J Bone Joint Surg [Am] 2008;90A:885-96

35. Farron A, Terrier A, Buchler P. Risks of loosening of a prosthetic glenoid implanted in retroversion. J Shoulder Elbow Surg 2006;15:521-6.

36. De Wilde L, Walch G. Humeral prosthetic failure of reversed total shoulder arthroplasty: a report of three cases. J Shoulder Elbow Surg 2006;15:260-4.

37. Livermore J, Ilstrup D, Morrey B. Effect of femoral head size on wear of the polyethylene acetabular component. J Bone Joint Surg [Am] 1990;72-A:518-28.

38. No authors listed. ASTM International. http://www.astm.org (date last accessed 1 June 2009).

39. Anglin C, Wyss UP, Pichora DR. Mechanical testing of shoulder prostheses and recommendations for glenoid design. J Shoulder Elbow Surg 2000;9:323-31.

40. Davis PR. Some significant aspects of normal upper limb functions. In: Engineers IoM, ed. Conference on joint replacement of the upper extremity. London, Institute of Mechanical Engineers, 1977.

41. Terrier A, Merlini F, Pioletti DP, Farron A. Total shoulder arthroplasty: downward inclination of the glenoid component to balance supraspinatus deficiency. J Shoulder Elbow Surg 2009; Epub.

42. Maxian TA, Brown TD, Pedersen DR, Callaghan JJ. Adaptive finite element modeling of long-term polyethylene wear in total hip arthroplasty. J Orthop Res 1996;14:668-75

43. Knight LA, Pal S, Coleman JC, et al. Comparison of long-term numerical and experimental total knee replacement wear during simulated gait loading. J Biomech 2007:40:1550-8. 